char xcters and production. We may mention, in passing, that the most recent work shows tneir probable identity with the precipitations, but not with the amboceptors, since a serum can be produced, after suitzble treatment, by $B$. pyocyaneus, which possesses immunizing but not agglutinating properties.

But in the practical application of serum diagnosis various phenomena may occur which must be known and understood in estimating the value of the results. In spite of early warnings, some at once pinned their faith on the supposed infallibility of the test, and then finding that it was unable to always determine an uncertain diagnosis, became unbelievers. The agglutinins, although not identical with the amboceptors, nevertheless go roughly together with them in their rate and conditions of production under ordinary circumstances. Consequently in very severe infections, when all the cells are hard hit, and many of them succumb, the agglutinin may either be used up or not formed, so that it is but natural for the reaction to be feeble or absent until the patient is already on the road to recovery. On the other hand, in mild attacks, which may be the result of either milder infections or of greater resistance, the reaction may naturally appear sooner and be stronger. Or, again, its absence may be due to an error in diagnosis. Finally, it may be the result of faulty technique.

The following points are worthy of consideration: Agglutinins may become converted into agglutinoids. These would in this form also unite with the bacilli like the complementoids with the amboceptor, but without producing any specific effect; no agglutination would take place. The bacilli may lose their agglutinability, although retaining the power of uniting with the agglutinin-namely, their agglutinable substance loses its functional atom group. This group is comparatively unstable, so that unsuitable culture media, etc. may make the bacilli nonagglutinable, although uniting with the agglutinin. If the serum contain both agglutinins and agglutinoids, and the latter have the greater affinity for the bacteria, even if present in less quantity, they may inhibit agglutination until the serum is so far diluted as to give the agglutinins a greater chance. This explains an early observation of my own for which I was then quite unable to account, that a higher dilution would sometimes agglutinate when lower one would not.

The agglutinable substance is partly soluble in $\mathrm{NaCl}$ solution, so that an emulsion of bacilli should always be made fresh, or the reaction becomes less sensitive.

\section{Partial Agglotinins.}

This expression is used to indicate the fact that several agglutinins may exist in a serum and may have slightly different cytophile groups, so that they will react differently as regards intensity with different strains of the same bacillus. Some may even act on nearly allied bacilii. Consequently it is a matter of importance what culture is used for serum diagnosis. It is also important in using an agglutinative serum, both for the identification of a bacillus and for a serum diagnosis, that the serum should be diluted as much as possible consistent with obvious action, since in this way the misleading action of the less numerous agglutinins is eliminated.

The serum reaction is also likely to be absent in cases of erroneous diagnosis. If bacteria are otherwise nearly allied it is, at any rate, possible that they may produce symptoms having much clinical resemblance. This seems to be so in typho-coloid fever. On the other hand the relationship between the bacteria may be so close that the patient's serum may act on each in comparatively low dilutions, and the various strains may be separable only by degrees of dilution. Of course, discretion and reason must be exercised in all these cases, so that serum diagnosis requires not only considerable technical knowledge and skill, but also an acquaintance with the clinical features of the case under investigation. It is only a symptom, and its value as such in any particular case will vary like the value of any other symptom.

There is a strange gap in our knowledge of etiology regarding those diseases of which one attack produces a very lasting immunity: of small-pox, typhus, scarlet fever, measles, and others we do not know the morbific germ. It is a curious coincidence that these diseases happen to be the most infec. tious. I believe that the discovery of their causes would throw great light on the phenomena of immunity. Even with our present knowledge I think we can assert that they are cases of mixed infection in which streptococci play a part, but only the minor part, as often in tubercle and diphtheria. Strepto- coccus scarlatinae seems to occur pretty constantly in the throat and in the blood of scarlet fever patients. In two cases of measles I have found in the heart blood at the necropsy a streptococcus which is always present in the throats of measles patients. But to my mind it is pretty certain that these are only accessory causes. For we know that immunity to streptococcus infection is short, and second attacks are common. Many people exposed to acarlet fever infection get sore throats repeatedly, but no scarlet fever; and streptococcus scarlatinae has been isolated from such cases.

The infectious fevers form an attractive theme for theorizing and a yet more attractive object for investigation. Their infectivity does not seem to be all of one kind. Thus yellow fever, an attack of which also confers a long immunity, has until quite recently been regarded as highly infectious. Yet the infection is certainly not by contagion; it is transmitted by the mosquito. The pathogenic micro-organism has not yet been found. Can it be that the infectious diseases of temperate climates are transmitted in a similar fashion? It seems possible, but unlikely.

Even so, we are not any nearer either the cause of the disease nor the reason for the long immunity. But I believe the discoverer of the cause will find with it the key to the innermost temple of the Theory of Immunity.

\section{ON THE \\ BACTERIOLYTIC POWER OF THE BLOOD, AND \\ ON ITS RELATION TO THE PROBLEMS OF ANTITYPHOID INOCULATION AND THE RECENT WORK OF DR. MACFADYEN.}

BY A. E. WRIGHT, M.D., Late Professor of Pathology, Army Medical School, Netley; Pathologist to

THE publication in the form of editorials in the last two numbers of the British Mrdical JournaL of the results of Dr. A. Macfadyen's researches on immunization against typhoid fever and the serumtherapy of the disease affords a convenient opportunity for bringing forward certain observations on the bacteriolytic power of the blood, and certain criticisms of Đr. Macfadyen's work. I may deal seriatim with the more important of the issues raised.

Problem of the Proper Constitution of the Vaccine.

In undertaking researches in connexion with the production of immunity against typhoid fever, the following propositions only can legitimately be taken as axiomatic:

1. The vaccine material must derive from a culture of the bacillus typhosus.

2. Owing to the risk which would attach to the employment of living cultures the vaccine material must consist either of a sterilized typhoid culture or of a constituent isolated from such a culture.

3. The vaccine material, however constituted, must be free from contaminating micro organisms.

Every other point in connexion with the constitution of the vaccine material is a debatable point, and deserves to be experimentally investigated. Among these points is, of course, the question as to whether the vaccine material I employ-which consists of agar or broth cultures sterilized at $60^{\circ}$ C. - constitutes the ideal vaceine material. It seems to me that the path of progress may possibly lie in eliminating from these cultures any poisonous element which may be found inert with respect to the production of immunizing substances. Reserving this point, I may here address mysel to the task of considering whether Dr. Macfadyen's vaccine material-constituted as it is by triturating typhoid bacteria at the temperature of liquid air-presents advantages over the ordinary sterilized culture which I employ.

ax In view of the results $I$ have obtained in the course of an experimental inquiry into the fate of typhoid bacteria introduced into the blood and lymph, it appears to me that the question I have raised above may be answered in the negative. I find that when an ordinary twenty-four-hour-old typhoid calture-supposing it to contain not more than about $\mathrm{I}, 000,000,000$ typhoid bacteria in the cubic centimetre-is mixed in a capillary tabe with an equal quantity of normal human serum or lymph (blister fluid), and is digested with this serum or lymph for thirty minutes at blood heat, considerably over 99 per cent. of the typhoid bacteria are dissolved by the 
serum. Where the experiment is carried out subsequent to inoculation with the typhoid vaccine I employ, a precisely similar effect may be obtained by the addition of one-fifth volume of serum or less to one volume of culture.

The technique employed is as follows:

Fitting a rubber teat on to a piece of glass tubing drawn out into a capillary stem, and placing a mark somewhere upon that stem, we draw up into the tube first one volume of a non-agglutinating human blood obtained from a prick in the finger, and then-keeping the successive volumes apart by bubbles of air-four volumes of an ordinary twenty-fourhour-old broth culture of the typhoid bacillus. Having intimately mixed the blood and the culture we place a drop of the mixture upon a carefully cleaned slide and draw it out into a thin film. The preparation is now stained with Leishman's stain or it is simply mounted in air under a coverglass. We then-employing an oil immersion lens and a high ocular-count the relative number of bacteria and red blood corpuscles on a succession of fields. Then taking the number of red blood corpuscles as corresponding to the normal of $5,000,000,000$ in the cubic centimetre, we calculate out from the ratio of red blood corpuscles to bacteria the number of micro-organisms in the cubic centimetre, taking into consideration the fact that four volumes of the broth culture have been mixed with a single volume of blood.* At the same time we take up into another capillary tube one volume of the serum which we desire to test and one volume of the typhoid culture. After mixing the contents of the tube we maintain them at a temperature of $37^{\circ} \mathrm{C}$. for half an hour. At the expiration of this period we take as before one volume of blood from the finger and mix this with four volumes of the digested serum and typhoid culture which have just been digested together. Preparing a film as before, we find on searching it with the oil immersion only a few isolated typhoid bacteria, instead of the many millions in the original film.

If we desire to arrive at the exact number of the bacteria which have survived, we employ the following procedure, which has been worked out by my friend Captain Stewart Douglas, I.M.S. We take an aliquot volume of the digested serum and culture, dilute it by mixing with it, by the technique referred to above, nine volumes of broth. Mixture having been effected, and a portion of the mixture having been reserved for further employment, we take a measured volume of our ten-fold dilution and spread it out over the surface of an agar tube of nutrient agar. To our reserved portion of tenfold dilution we now add nine further volumes of broth, and we again plant out upon agar a measured volume of the hundred-fold dilution thus obtained.: We then place the agar tubes in the incubator, count the number of colonies which develop, and calculate from these data the number of surviving bacteria. $t$

The following figures, which represent the numbers of bacteria dealt with and the number of survivors found in the case of three successive experiments instituted by Captain Douglas with typhoid cultures and normal human serum, will give an idea of the magnitude of the forces of dissolution which come into play:

\begin{tabular}{|c|c|c|}
\hline- & $\begin{array}{l}\text { Number of;Typhoid Bacteria } \\
\text { contained per c.cm. in } \\
\text { the Culture Employed. }\end{array}$ & $\begin{array}{l}\text { Number of Survivors per } \\
\text { c.cm. after Digesting } \\
\text { r Vol. of Culture with I Vol. } \\
\text { of Serum at } 37^{\circ} \mathrm{C} \text {. } \\
\text { for half an hour. }\end{array}$ \\
\hline Experiment I ... & $1,100,050,000$ & $\mathrm{I} 4 \mathrm{O}, \mathrm{CcO}$ \\
\hline Experiment $2 \ldots$ & $x, \mathrm{CO}_{5}, 000,000$ & 144,000 \\
\hline Experiment $_{3}$.. & $\mathrm{I}, 050,000, \mathrm{c00}$ & $156,0=0$ \\
\hline
\end{tabular}

It appears from the above that the trituration of the typhoid bacteria at the temperature of liquid air which constitutes Dr. Macfadyen's technical innovation effects only what is effected spontaneously in the organism when the typhoid bacillus is inoculated. It may be taken that the I,000,000,000 typhoid bacteria which I administer as a first dose of typhoid vaccine would dissolve in little more than $1 \mathrm{c.cm}$. of the patient's lymph.

It may suggest itself that even if the process of triturating the bacteria be surplusage from the point of view of obtaining the constituents of the typhoid bacteria in the form of a solution, it may be incidentally of advantage if it avoids the need for the sterilization of the vaccine material by heat. I cannot, however, in view of the difficulty which has been experienced in effecting the sterilization of tubercle cultures by trituration, suppose that a process of after-sterilization would be dispensed with in the case where a typhoid vaccine was to be employed upon man; and I assume, in view of the risks of contamination which are presumably associated with the mechanical operations involved in $\mathrm{Dr}$. Macfadyen's triturating operation, that exposure to a * For further details of the enumeration procedure vide Lancet, July ${ }_{5}$ th,

† The automatic pipette and the procedure figured and described by me in the Iancet, July 5 th and December 6 th, r902, will be found to furnish convenient means for calibrating the measuring pipette. temperature of $60^{\circ} \mathrm{C}$. for ten minutes would be quite insufficient for sterilization. The commentary will suggest itself.

Standardization of the Vaccine.

Of dominating importance in connexion with every inoculation process is the standardization of the vaccine. That standardization involves, on the one hand, the determination of the amount of the immunizing element contained in a standard volume of the vaccine material ; on the other hand, the adjustment of the dose administered to the resisting powers of the man or animal subjected to the inoculation.

Upon the assumption, which I make in common with Dr. Macfadyen, that a constituent of the bacterial protoplasm constitutes the essential immunizing element, it will be the amount of that protoplasm in the vaccine which will come into consideration in appraising the potency of the vaccine. The amount of that protoplasm can be estimated by two alternative methods. We may either, dealing with a definite volume of culture, enumerate the bacteria; or we may filter off these bacteria, wash on the filter, collect, dry, triturate, weigh, and then add-as is done in the preparation of anti-tubercle vaccine (tuberculin) - to a definite weight of the triturated bacteria a measured volume of fluid I have adopted the former method, that is, the method of enumerating the bacteria which furnish the bacterial protoplasm. And I have reason to be satisfied with the results, now that $I$ am in poseession of the method of enumeration referred to above, which takes account of dead as well as living bacteria. Dr. Macfadyen has adopted-with the difference that he triturates his bacteria for some reason unrevealed at the temperature of liquid air-the method employed in the case of the preparation of tuberculin. I do not, in the light of experience gained in sending out typhoid vaccine in the mass, begrudge him his more complicated procedure.

Adjustment of the Dose of Typhoid Vaccine to the Resisting Powh of the Organism subjected TO THE INOCULATION.

The important issues that are involved in the correct regulation of the dose must in this connexion be clearly appreciated. It was first brought out perfectly clearly by Ehrlich in connexion with the inoculation of tetanus toxin, afterwards by Madsen in connexion with the inoculation with diphtheria toxin, that after the inoculation of bacterial toxins into the system there is developed a phase of diminished resistance, followed by a phase of increased resistance dependent upon an elaboration of untitoxins in the organism. The same succession of what I have called "negative" and "positive" phases has been shown to obtain, in connexion with all other inoculation processes which have been investigated. I myself have shown that this law of immunization has a wider application than had been previously discerned. I have demonstrated, ${ }^{*}$ in connexion with the therapeutic inoculation of staphylococcus cultures into man that we have here to deal with a negative followed by a positive phase of a phagocytic power, and in the case of antityphoid inoculation ${ }^{2}$ in man and animals that we have to deal with a negative followed by a positive phase of the bactericidal power. I have pointed out ${ }^{3}$ also that the negative phase may be unduly prolonged, and the prophylactic effects of an inoculation imperilled where excessive doses of bacterial vaccines are inoculated.

Having recognized the general issues involved in the adjustment of the dose of a bacterial vaccine, it would seem that where the application of a typhoid vaccine to man is in contemplation, the experiments which are required ought to be undertaken not on animals but on man himself. I have endeavoured to point the way by devising and applying methods ${ }^{4}$ which permit of the effects of a bacterial inoculation being investigated on man in an accurately quantitative manner.

If Dr. Macfayden has not followed this lead in testing the effect of his vaccine material I do not doubt that it is because he assumed that his mechanical operations placed in his hands a vaccine material which was entirely sui generis, and which caused the elaboration of an entirely novel series of immunizing substances in any organism that he might choose to subject to inoculation. It is claimed by or for Dr. Macfayden that he has discovered (induced) the elaboration of a novel element, to wit, of a typhoid antitoxin in the blood of his inoculated animals.

I have above expressed the opinion that there is nothing novel in the vaccine material devised by Dr. Macfadyen except that it was prepared with liquid air. I am further of opinion 
that we have as yet no reason to conclude that any novel immunizing element has been developed by the inoculation of this vaccine material. When we have realized that an inoculation of a moderate quantity of a typhoid culture is by reason of the dissolution of the bacteria in the fluids of inoculated organism the equivalent of the introduction of a quantum of poisonous bacterial protoplasm in a dissolved form, and when we remember that it has been established in very numerous experiments that a normal animal treated with serum from an inoculated animal will survive the inoculation of an otherwise lethal dose of typhoid culture, we may, it seems to me, infer the presence of antitoxins in the serum which has exerted the therapeutic effect. Even if a difficulty of interpretation "here arises in consequence of the complexity of the conditions, all doubt with regard to the elaboration of an antitoxin in the human system after ordinary typhoid inoculation is dispelled by the fact that in every case where a proper interval has been observed between first and second inoculation the symptoms are much slighter after a second inoculation undertaken with a larger dose, than after the first inoculation undertaken with a smaller dose.

Seruntherapy OF TyphoId.

may turn to the question of the serumtherapy of 1 yphoid fever. It has been claimed by or for Dr. Macfadyen that an important step has been taken towards the solution of the problem of the serumtherapy of typhoid fever by the work which has just been passed in review. Even if it had been established - and I have contended that it has not been established-that Dr. Macfadyen has devised a novel vaccine material which induced the elaboration of a novel antitoxic element in the inoculated organism, the problem of serumtherapy would not necessarily have been brought any nearer to a practical solution. Those engaged in medical research must, under peril of keeping the word of promise to the ear only to break it to the hope, emphasize in season and out of season that there is absolutely no outlook for the successful practical exploitation of an antitoxic serum against any disease unless, as obtains in the case of antidiphtheria serum the quantity of antitoxin contained in the few cubic centimetres of foreign blood which can be inoculated into the patient is sufficiently considerable to neutralize at least a substantial fraction of the bacterial toxin generated in his system.

Unless Dr. Macfadyen's unpublished experiments show that the production of typhoid antitoxin in the system of his monkeys is of this order of magnitude-and the absence of all quantitative data from his publications gives occasion for an anxiety on this point-and unless further he has ascertained that an antitoxin formation on this scale can be induced in an animal capable of furnishing a sufficient supply of serum -and the unsuccessful experiments made up to the present on horses, asses, sheep, and goats here dash our hopes-the prospects of serumtherapy for typhoid seem to me to stand at present just where they stood before the announcement of the results of Dr. Macfadyen's work.

$$
\text { REFERENCES. }
$$

1 On the Treatment of Furunculosis, Sycosis, and Acne by the Inocula tion of a Staphylococcus Vaccine, Lancet. March 29 th, 1902. 2 On the Changes. Effected by Antityphoid Inoculation in the Ractericidal Powe of Human Hlood, Lancet, September $x_{4}$ th, rgor: On the Bactericidal Powe of the Blood. Jnl. of Hygiene, October, 1902. 3 Loc cit. 4 Lancet, July 5 th, ¿9o2; Proc. Roy. Soc., vol. lxxi.

\section{MEMORANDA:}

\section{MEDICAL, SURGICAL, OBSTETRICAL, THERA- PEUTICAL, PATHOLOGICAL, ETc.}

HEAD DROP FOLLOWING DIPHTHERIA.

IN reference to the interesting memorandum by Dr. Sharp in the British Medical Journal of February 7 th on a case of paresis of the retro-cervical muscles following diphtheria, I would like to record a case which I have recently met with.

E. S., aged 6, was admitted into this hospital on November 28th, 1902, notified as diphtheria. I was also notified by telephone that the case required immediate attention, which $I$ found to be the case, and I was obliged to open the trachea with very little preparation or cleansing, as the child's face was becoming black. After the tracheotomy the culour of the child soon became normal, and I injected beneath the skin of the flank 6,000 units of antitoxin. The child appeared to be doing well, and could do without the tube at the end of three dass, although in the meantime she had coughed up sufficient membrane to almost half fill an ordinary test tube.

On the fifth night I was called up to see the child (whom I had seen on my night visit to the ward to be sleeping oalmly), and was informed by the rurse that the child had jumped up in bed, and, after gasping for breath and tugging at her throat, had dropped back pulseless and breathless. I found the child with a very feeble, rapid pulse, and very shallow. slow respirations. I gave her a subcutaneous injection of 5 minims of liquor strychninae, and as she improved I went back to bed. In twenty minutes I was again called to see the child, and $I$ found her twitching very badly about the face and arms, the effect apparently of the strychnine, although the dose was not large. The pulse and respiration had improved, but as the spasmodic twitching did not improve I gave her a few whiffs of chloroform, during which the spasm subsided, and the child went to sleep and passed a very good night.

She continued to improve steadily for three weeks, when we first noticed that she had a nasal intonation of her voice, and a little fluid returned through the nose when drinking, that she was less able to bear the weight of her body upon her legs, and that she had trouble in raising her head and keeping it raised. The dropping of the head got worse until she seemed to have no control over it, and when raised and let go it fell upon the chest and towards the left shoulder. The legs got a little stronger but she could not bear her weight upon them.

The nasal intonation of voice and return of fluid through the nose got better, and it was in this condition that she was sent home after forty-six days in hospital.

On the eighteenth day after discharge she was notified and came in with small-pox, pretty full but discrete, probably being infected at home from a case we received from the same house about five days after her return home. To my surprise the child could bear her weight on her legs and could walk. and could do practically any movement with the head, and showed no sign of head drop, nor would a casual observer have detected anything wrong: still there was slight weakness of the neck muscles. She is now quite convalescent, and has lost all trace of weakness of the part, can run about and is bright and intelligent, and is about to be discharged. It is probable that these cases are sometimes overlooked, more especially in infants, in whom the head is for some time after birth more or less uncontrolled. The paralysis, if slight, may also escape notice in older children. I can recall two instances of the kind which would have probably escaped my observation had not my attention been called to them by the sister of the ward.

$$
\begin{gathered}
\text { JosEPH BEARD, F.R.C.S.Ed., L.R.C.P.Lond., } \\
\text { Resident Medical Superintendent, Corporation Hospital, } \\
\text { Bootle. }
\end{gathered}
$$

\section{DISAPPEARANCE OF AN APPARENTLY MALIGNANT} VAGINAL GROWTH.

LAST March I was asked to see a lady, aged 73, who had been suffering for some weeks from haemorrhage and discharge from the vagina, accompanied by a good deal of pain. She had lost flesh and become weak and anaemic, and the frequently recurring haemorrhage practically confined her to the house. She had suffered from piles for some years previously, and could never get an action of the bowels without an enema.

Upon examination I found a growth occupying the vagina and involving the cervix uteri and the posterior and anterior wall of the vagina to within an inch of the vulva. It was soft and friable and bled very readily, and there was a fetid discharge.

I had no doubt whatever that it was a cancerous growth and that it was too extensive to be capable of removal. The friends desired another opinion, and Sir John Williams was called in consultation. He corroborated my view of the case, and also felt no doubt as to the nature of the growth.

The patient was ordered an injection of chinosol for vaginal irrigation, and to keep the recumbent position for the greater part of the day, and a laudanum and starch enema to be given every night to ease the pain.

In October I was told she was much better and that the haemorrhage and discharge had ceased, together with the pain. I saw her in November, and was astonished to find the growth gone. There was a slight cicatricial contraction of the vagina below the cervix, but the mucous membrane was smooth, the uterus was freely movable, and the patient declared herself quite well again. There is still a pile which 